\title{
Computing the real zeros of hypergeometric functions
}

\author{
Amparo Gil ${ }^{\mathrm{a}}$, Wolfram Koepf ${ }^{\mathrm{b}}$ and Javier Segura ${ }^{\mathrm{a}}$ \\ ${ }^{a}$ Depto. de Matemáticas, Estadística y Computación, Universidad de Cantabria, 39005-Santander, Spain \\ E-mail: \{amparo.gil;javier.segura\}@unican.es \\ ${ }^{b}$ Universität Kassel, FB 17 Mathematik-Informatik, 34132-Kassel, Germany \\ E-mail: koepf@mathematik.uni-kassel.de
}

Received 2 December 2003; accepted 18 February 2004

Communicated by S. Paszkowski

\begin{abstract}
Efficient methods for the computation of the real zeros of hypergeometric functions which are solutions of second order ODEs are described. These methods are based on global fixed point iterations which apply to families of functions satisfying first order linear difference differential equations with continuous coefficients. In order to compute the zeros of arbitrary solutions of the hypergeometric equations, we have at our disposal several different sets of difference differential equations (DDE). We analyze the behavior of these different sets regarding the rate of convergence of the associated fixed point iteration. It is shown how combinations of different sets of DDEs, depending on the range of parameters and the dependent variable, is able to produce efficient methods for the computation of zeros with a fairly uniform convergence rate for each zero.
\end{abstract}

Keywords: zeros, hypergeometric functions, fixed point iterations, numerical algorithms

AMS subject classification: $33 \mathrm{CXX}, 65 \mathrm{H} 05$

\section{Introduction}

The zeros of hypergeometric functions are quantities which appear in a vast number of physical and mathematical applications. For example, the zeros of classical orthogonal polynomials (OP) are the nodes of Gaussian quadrature; classical OP (Hermite, Laguerre and Jacobi polynomials) are particular cases of hypergeometric functions. Also, the zeros of Bessel functions and their derivatives appear in many physical applications and there exists a variety of methods of software for computing these zeros.

However, an efficient algorithm which can be applied to the computation of all the zeros of any hypergeometric function in any real interval (not containing a singular point of the defining ODE) is still missing.

In $[3,7]$ methods were introduced which are capable of performing this task for hypergeometric functions which are solutions of a second order ODE; an explicit Maple algorithm was presented in [4]. The starting point of the methods is the construction of 
a first order system of differential equations

$$
\begin{aligned}
y^{\prime}(x) & =\alpha(x) y(x)+\delta(x) w(x), \\
w^{\prime}(x) & =\beta(x) w(x)+\gamma(x) y(x),
\end{aligned}
$$

with continuous coefficients $\alpha(x), \beta(x), \gamma(x)$ and $\delta(x)$ in the interval of interest, relating our problem function $y(x)$ with a contrast function $w(x)$, whose zeros are interlaced with those of $y(x)$. Typically, the contrast function $w(x)$ satisfies a second order ODE similar to the second order ODE satisfied by the problem function.

Given a hypergeometric function $y(x)$ there are several known options to choose a contrast function $w(x)$. As an example, considering a Jacobi polynomial

$$
y(x)=P_{n}^{(\alpha, \beta)}(x)=\frac{(\alpha+1)_{n}}{n !}{ }_{2} \mathrm{~F}_{1}\left(-n, n+\alpha+\beta+1 ; \alpha+1 ; \frac{1-x}{2}\right)
$$

we could take as contrast function $w_{\mathrm{OP}}(x)=P_{n-1}^{(\alpha, \beta)}(x)$ but also $w_{\mathrm{D}}(x)=(\mathrm{d} / \mathrm{d} x) P_{n}^{(\alpha, \beta)}(x)$ is a possible choice. Both contrast functions are ${ }_{2} \mathrm{~F}_{1}(a, b ; c ; x)$ hypergeometric functions with parameters $a, b, c$ differing by integer numbers from the parameters of the problem function $y(x)$.

When the contrast function in the previous example is $w_{\mathrm{OP}}(x)=P_{n-1}^{(\alpha, \beta)}(x)$ the first order differential system is related to the three term recurrence relation for Jacobi polynomials. It may seem that this is a natural differential system to consider. However, it was numerically observed that the fixed point method which can be obtained from this differential system becomes relatively slow for the zeros of $P_{n}^{(\alpha, \beta)}$ close to \pm 1 [4]. Because the extreme zeros approach to \pm 1 as $n \rightarrow+\infty$, the efficiency for the computation of such zeros decreases as the order increases. Similar problems arise, for example, when $\alpha \rightarrow-1^{+}$or $\beta \rightarrow-1^{+}$. In fact, the number of iterations required to compute the extreme zeros tend to infinity in these limits. Similar problems take place for Laguerre polynomials $L_{n}^{\alpha}(x)$ for the smallest (positive) zero. Fortunately we will later show how the selection of $w_{\mathrm{D}}(x)$ as contrast function gives a much better asymptotic behavior for the resulting fixed point iteration for the extreme Jacobi zeros. For the Laguerre case, a similar solution is possible.

These two examples illustrate the need to analyze the convergence of the resulting fixed point iteration for the different available contrast functions. Although for any adequate contrast function (satisfying the necessary conditions [3,7]) the resulting fixed point method is quadratically convergent, the non-local behavior of the method and the corresponding estimation of first guess values for the zeros may result in disaster for certain contrast functions in some limits. As a result of this study, we will obtain explicit methods for the computation of the real zeros of hypergeometric functions with a good asymptotic behavior and a fairly uniform convergence rate in the whole range of parameters. 


\section{Theoretical background}

Let us now briefly outline the main ingredients of the numerical method. For more details we refer to [3,7]. It was shown in $[3,7]$ that, given a family of functions $\left\{y_{k}^{(1)}, y_{k}^{(2)}\right\}$, depending on one parameter $k$, which are independent solutions of second order ODEs

$$
y_{k}^{\prime \prime}+B_{k}(x) y_{k}^{\prime}+A_{k}(x) y_{k}=0, \quad k=n, n-1,
$$

and satisfy relations of the type:

$$
\begin{aligned}
y_{n}^{\prime} & =a_{n}(x) y_{n}+d_{n}(x) y_{n-1}, \\
y_{n-1}^{\prime} & =b_{n}(x) y_{n-1}+e_{n}(x) y_{n}
\end{aligned}
$$

the coefficients $a_{n}(x), b_{n}(x), d_{n}(x), e_{n}(x), B_{k}(x)$ and $A_{k}(x)$ being continuous and $d_{n} e_{n}<0$ in a given interval $\left[x_{1}, x_{2}\right]$, fixed point methods (equation (7)) can be built to compute all the zeros of the solutions of (3) inside this interval. These differencedifferential equations (4) are called general because they are satisfied by a basis of solutions $\left\{y_{k}^{(1)}, y_{k}^{(2)}\right\}$. The fact that the DDEs are general and with continuous coefficients in an interval $I$ implies [7] that $d_{n} e_{n} \neq 0$ in this interval. Conversely, given $\left\{y_{n}^{(i)}, y_{n-1}^{(i)}\right\}$, $i=1,2$, independent solutions of the system (4) and $d_{n} e_{n} \neq 0$, then $\left\{y_{k}^{(1)}, y_{k}^{(2)}\right\}$, $k=n, n-1$, are independent solutions of the ODEs (3). The method can then be applied to compute the zeros of any solution of such ODEs.

It was shown that the ratios $H_{i}(z)(i= \pm 1)$ :

$$
\begin{aligned}
H_{i}(z) & =-i \operatorname{sign}\left(d_{n_{i}}\right) K_{n_{i}} \frac{y_{n}(x(z))}{y_{n+i}(x(z))}, \\
K_{n_{i}} & =\left(-\frac{d_{n_{i}}}{e_{n_{i}}}\right)^{i / 2}, \quad z(x)=\int \sqrt{-d_{n_{i}} e_{n_{i}}} \mathrm{~d} x,
\end{aligned}
$$

where $n_{+1}=n+1, n_{-1}=n$, satisfy the first order equations

$$
\dot{H}_{i}(z)=1+H_{i}(z)^{2}-2 \eta_{i}(x(z)) H_{i}(z),
$$

where

$$
\eta_{i}(x)=i \frac{1}{\sqrt{-d_{n_{i}} e_{n_{i}}}}\left(a_{n_{i}}-b_{n_{i}}+\frac{1}{2}\left(\frac{e_{n_{i}}^{\prime}}{e_{n_{i}}}-\frac{d_{n_{i}}^{\prime}}{d_{n_{i}}}\right)\right),
$$

and the dot means derivative with respect to $z$ while the prime is the derivative with respect to $x$. Using equation (6), one can show that

$$
T_{i}(z)=z-\arctan \left(H_{i}(z)\right)
$$

are globally convergent fixed point iterations (FPI): given a value $z_{0}$ between two consecutive zeros $\left(z_{n_{i}}^{1}, z_{n_{i}}^{2}\right)$ of $y_{n_{i}}(x(z))$ (consecutive singularities of $H_{i}(z)$ ), the iteration of (7) converges to $z_{n} \in\left(z_{n_{i}}^{1}, z_{n_{i}}^{2}\right)$, where $x_{n}=x\left(z_{n}\right)$ is a zero of $y_{n}(x)$. 
Global bounds for the distance were provided which lead to iteration steps that can be used to compute new starting values for obtaining all the zeros inside a given interval.

It was shown that, in intervals where $\eta_{i}$ does not change sign, either

$$
\left|z\left(x_{n_{i}}^{1}\right)-z_{n}\right|<\frac{\pi}{2} \quad \text { and } \quad\left|z\left(x_{n_{i}}^{2}\right)-z_{n}\right|>\frac{\pi}{2}, \quad \eta_{i}>0 \text {, }
$$

or

$$
\left|z\left(x_{n_{i}}^{1}\right)-z_{n}\right|>\frac{\pi}{2} \quad \text { and } \quad\left|z\left(x_{n_{i}}^{2}\right)-z_{n}\right|<\frac{\pi}{2}, \quad \eta_{i}<0 .
$$

In this way $\pi / 2$ is the choice for the iteration step when the second situation takes place $\left(\eta_{i}<0\right)$; this means that if $y_{n_{i}}(x(z))$ has at least a zero larger than $z_{n}$, then

$$
\lim _{j \rightarrow \infty} T^{(j)}\left(z_{n}+\frac{\pi}{2}\right)
$$

is the smallest zero larger than $z_{n}$. Similarly, when $\eta_{i}>0$, the iteration step will be $-\pi / 2$ instead of $\pi / 2$ (backward sweep). When $\eta_{i}$ changes sign, forward and backward schemes can be combined [3].

As shown in [3], one can find a new set of functions $\tilde{y}_{k}(z) \equiv \lambda_{k}(z) y_{k}(x(z))$, with $\lambda_{k}(z)$ having no zeros, such that the ratio $H_{i}$ can be written as $H_{i}(z)=\tilde{y}_{n}(z) / \tilde{y}_{n+i}(z)$ and such that $\tilde{y}_{n}(z)$ and $\tilde{y}_{n+i}(z)$ satisfy second order ODEs in normal form:

$$
\frac{\mathrm{d}^{2} \tilde{y}_{n}}{\mathrm{~d} z^{2}}+\tilde{\mathrm{A}}_{n} \tilde{y}_{n}=0, \quad \frac{\mathrm{d}^{2} \tilde{y}_{n+i}}{\mathrm{~d} z^{2}}+\tilde{\mathrm{A}}_{n_{i}} \tilde{y}_{n+i}=0,
$$

where

$$
\tilde{\mathrm{A}}_{n}(z)=1+\dot{\eta}_{i}-\eta_{i}^{2}, \quad \tilde{\mathrm{A}}_{n_{i}}(z)=1-\dot{\eta}_{i}-\eta_{i}^{2} .
$$

Of course, the functions $\tilde{y}_{k}(z)$ and $y_{k}(x(z))$ have the same zeros because the functions $\lambda_{k}(z)$ have no zeros.

Finally, we recall that using monotony conditions of $A_{n}(z)$ the iteration steps $\pm \pi / 2$ (equation (10)) can be improved according to [3, theorem 2.4]:

Theorem 2.1. If $z_{-1}<z_{0}<z_{1}$ are three consecutive zeros of $y_{n}(x(z))$ and $\eta_{i}(z) \times$ $\dot{\tilde{\mathrm{A}}}_{n}(z)>0$ in $\left(z_{-1}, z_{1}\right)$ then $z_{j}=\lim _{n \rightarrow \infty} T^{(n)}\left(z_{0}+\Delta z_{0}\right)$, where $\Delta z_{0}=z_{0}-z_{-j}$, $j=\operatorname{sign}(\eta)$. The convergence is monotonic.

\subsection{Oscillatory conditions}

We are interested in computing zeros of oscillatory solutions of second order ODEs and, in particular, on building algorithms for the computation of the zeros of the hypergeometric functions. If a second order differential equation has a given number of singular regular points, we divide the real axis in subintervals determined by the singularities and search for the zeros in each of these subintervals. We only apply the algorithms if it is not disregarded that the function can have two zeros at least in the subinterval under consideration. 
We consider that an ODE has oscillatory solutions in one of these subintervals if it has solutions with at least two zeros in this subinterval; otherwise, if all the solutions have one zero at most we will call these zeros isolated zeros. The fixed point methods (FPMs) before described deal with the zeros of any function satisfying a given differential equation, no matter what the initial conditions are on this function. Isolated zeros for a given solution depend on initial conditions or boundary conditions for this solution and are, in any case, easy to locate and compute.

There are several ways to ensure that a solution $y_{n}(x)$ has at most one zero in an interval; among them:

Theorem 2.2. If one of the following conditions is satisfied in an interval $I$ (where all the coefficients of the DDEs are continuous) then $y_{n}$ and $\left(y_{n_{i}}\right)$ have at most one zero in the interval $I$ (trivial solutions excluded):

1. $d_{n_{i}}(x) e_{n_{i}}(x) \geqslant 0$ in $I$ [7].

2. $\left|\eta_{i}(x)\right| \geqslant 1$ in $I[3]$.

3. $\tilde{\mathrm{A}}_{n}<0\left(\tilde{\mathrm{A}}_{n_{i}}<0\right)[3]$.

The condition $e_{n_{i}} d_{n_{i}}<0$ is required for the method to apply. Furthermore, it is known that when the DDEs (4) are general, $d_{n_{i}} e_{n_{i}}$ can not change sign. Therefore $d_{n_{i}} e_{n_{i}}<0$ is a clear signature for the oscillatory character of the differential equation.

\subsection{Hypergeometric functions; selection of the optimal DDEs}

For hypergeometric functions several DDEs are available for the construction of fixed point iterations (FPIs), depending on the selection of contrast function. equation

Let us start by considering, for example, the case of the confluent hypergeometric

$$
x y^{\prime \prime}+(c-x) y^{\prime}-a y=0 .
$$

One of the solutions of this differential equation are Kummer's series

$$
M(a, c, x) \equiv{ }_{1} \mathrm{~F}_{1}(a ; c ; x)=\sum_{k=0}^{\infty} \frac{(a)_{k}}{(c)_{k} k !} x^{k},
$$

for which different difference-differential relations are available. Indeed, denoting $\alpha_{n}=$ $\alpha+k n, \gamma_{n}=\gamma+m n$ and $y_{n} \equiv M\left(\alpha_{n}, \gamma_{n}, x\right)$ we will have different sets of DDEs (equation (4)) for different selections of $(k, m)$.

For Gauss hypergeometric functions ${ }_{2} \mathrm{~F}_{1}(a, b ; c ; x)$, which are solutions of the ODE

$$
x(1-x) y^{\prime \prime}+[c-(a+b+1) x] y^{\prime}-a b y=0,
$$

the possible DDEs are determined by three-vectors with integer components, that is, we will consider $y_{n} \equiv{ }_{2} \mathrm{~F}_{1}(\alpha+k n, \beta+\ln ; \gamma+m n ; x)$ and the associated DDEs will be 
named $(k, l, m)$-DDEs. Finally, for the case of the hypergeometric functions ${ }_{0} \mathrm{~F}_{1}(; c ; x)$, we can only consider families $y_{n}={ }_{0} \mathrm{~F}_{1}(; \gamma+k n ; x)$ and the different relations are described by the integer numbers $k$.

Our FPMs can only be applied to solutions of second order ODEs. This restricts our study to the hypergeometric functions ${ }_{0} \mathrm{~F}_{1}(; c ; x),{ }_{2} \mathrm{~F}_{0}(a, b ; ; x),{ }_{1} \mathrm{~F}_{1}(a ; c ; x)$ and ${ }_{2} \mathrm{~F}_{1}(a, b ; c ; x)$.

Regarding the selection of the different DDEs available, we will restrict ourselves to:

1. DDEs with continuous coefficients except at the singular points of the defining differential equations.

2. The most simple DDEs in a given recurrence direction which allow the use of improved iteration steps. Taking as example the case of confluent hypergeometric functions, this means that the $(1,0)$-DDE will be described, and the analysis of the $(-1,0),(2,0), \ldots$ DDEs will be skipped.

The first restriction is convenient for simplicity and it means that the problem function and the contrast function have zeros interlaced in each subdivision of the real interval defined by the singular points of the differential equation; this is a convenient property for a simple application of the FPMs and enables the application of each DDE to compute all the zeros in the different subintervals of continuity of the solutions of the differential equation.

Regarding the second restriction, and considering the case of confluent hypergeometric functions as example, it should be noted that for the $(k, m)$-DDE, generally two FPIs are available, one of them based on the ratio $H_{-1}=y_{n}(x) / y_{n-1}(x)$ and a second one based on $H_{+1}(x)=y_{n}(x) / y_{n+1}(x)$. As described in [3], generally one of these two iterations is preferable because improved iteration steps can be considered according to theorem 2.1 (theorem 2.4 in [3]). If we considered the $(-k,-m)$-DDE, the two associated ratios $H_{i}, i= \pm 1$, would be the same as before (replacing $i$ by $-i$ ). Because both selections of DDEs are equivalent, only one of them will be discussed. By convention, we will consider pairs $(k, m)$ for which the iteration on $H_{-1}$ can take advantage of the monotony property of $\hat{\mathrm{A}}_{n}(x(z))$, as described in [3], for classical orthogonal polynomial cases (Jacobi, Hermite, Laguerre). Once we have fixed this criterion the index $i$ in equations (6)-(12) can be dropped. We consider the following additional notation: given a vector $\vec{u}$ with integer components, we will denote by $\operatorname{DDE}(\vec{u})(\operatorname{FP}(\vec{u}))$ the corresponding DDE (FPI) based on the ratio $y_{n} / y_{n-1}$ for $x>0$.

On the other hand, and considering the confluent case as illustration, we will not analyze $\operatorname{DDE}(2,2)$ nor any successive multiples of the $\operatorname{DDE}(1,1)$. This is so because the first restriction is generally violated if successive multiples of a DDE are considered (there are exceptions to this; see the case of ${ }_{0} \mathrm{~F}_{1}$ hypergeometric functions). 


\subsubsection{Selection of the optimal DDEs}

There are several (and related) criteria to select among the available DDEs to compute the zeros of a given function $y$. The associated FPMs tend to be more efficient as we are closer to any of the following two situations:

1. $\eta(x)=0$,

2. the coefficient $\tilde{\mathrm{A}}_{n}$ is constant.

Of course, the first condition implies the second one (see equations (11) and (12)). The first condition makes the FPI converge with one iteration for any starting value. The second condition makes the method an exact one using improved iteration steps (there is even no need to iterate the FPM).

Let us recall that the FPIs associated to a given system of DDEs are quadratically convergent to a zero $z_{0}$ (in the transformed variable $z$ ) with asymptotic error constant $\eta\left(x\left(z_{0}\right)\right)$. Therefore, the smaller $|\eta(x)|$ is the fastest the convergence is expected to be, at least for starting values close enough to $z_{0}$. On the other hand, the smaller the absolute value of variation of $\tilde{\mathrm{A}}_{n}$ is, the better the improved iteration (theorem 2.1) will work because this implies the exactness of the iteration criteria to estimate starting values from previously computed zeros. This second criterion (on the variation of $\tilde{\mathrm{A}}$ ) is more difficult to apply, as we will later see. It is, however, more relevant to improve the iterative steps for obtaining starting values to compute zeros than to improve the local convergence properties of the fixed point methods, which are quadratically convergent anyway.

Indeed, as was described in [4], the natural FPIs for orthogonal polynomials of confluent hypergeometric type $(\mathrm{FP}(-1,0))$ tend to converge slowly for the computation of the first positive zeros when they become very small. This, for instance, is the case for Laguerre polynomials $L_{n}^{\alpha}(x)$ when $\alpha \rightarrow-1^{+}$. The reason for this behavior lies in the fact that the associated change of variables is singular at $x=0$ :

$$
z=\sqrt{(b-a)(1-a)} \ln x .
$$

In this way, the interval of orthogonality for the Laguerre polynomials $(0,+\infty)$ is transformed into $(-\infty, \infty)$ in the $z$ variable. This means that the zeros which are very small in the $x$ variable, tend to go to $-\infty$ in the $z$ variable. Therefore after computing the second smallest zero, $x_{2}$, the next initial guess for the FPI, $z\left(x_{2}\right)-\pi / 2$, may lie well far apart for the value $z\left(x_{1}\right), x_{1}$ being the smallest zero. Although it is guaranteed that the FPI will converge to $z\left(x_{1}\right)$, it could take a considerable number of iterations to approach this value.

Fortunately, we will see that the rest of FPMs (different to $\operatorname{FP}(k, 0)$ ) do not show such a singularity; therefore, we expect better behavior near $x=0$ for these iterations.

This suggests that, given two FPIs with associated change of variables $z_{1}(x), z_{2}(x)$ respectively, one should choose that one which gives the largest displacement in the $x$ variable for the same step in the corresponding $z$ (the typical value being $\pi / 2$ ). Let us stress that the possibility of passing the next zero is ruled out: in the algorithms the 
sequence of all $z$ values calculated in a backward (forward) sweep form monotonically decreasing (increasing) sequences.

We will therefore say that the change of variables $z_{1}$ behaves better than $z_{2}$ if $x\left(z_{1}+\right.$ $\Delta z)>x\left(z_{2}+\Delta z\right)$, for a typical value of $\Delta z(\approx \pi / 2)$. Given the definition of the changes of variables $z(x)$ :

$$
x(z+\Delta z)-x(z)=\int_{z}^{z+\Delta z} \frac{1}{\tilde{d}_{n}(x(z))} \mathrm{d} z,
$$

where $\tilde{d}_{n}=\sqrt{-d_{n} e_{n}}$, we can say that the change of variable $z_{1}(x)$ (and its associated FPI) is more appropriate than the change $z_{2}(x)$ when its coefficient $\tilde{d}_{n}=\sqrt{-d_{n} e_{n}}$ is smaller than the corresponding coefficient for $z_{2}(x)$.

Therefore, an alternative non-local prescription to that one dealing with $\tilde{\mathrm{A}}_{n}$ is the following: among the possible DDEs and associated fixed point iterations, choose that one for which $\left|d_{n} e_{n}\right|$ is smallest. As we will see, this is an easy to apply criterion which correctly predicts the more appropriate DDEs and FPI depending on the range of the parameters and the dependent variable.

\section{Analysis of hypergeometric functions}

We will use the DDEs satisfied by hypergeometric series as generated by the Maple package hsum.mpl [5]. The results for each of the family of functions (change of variable $z(x)$, function $\eta(x)$, etc.) considered can be automatically generated using the package zeros.mpl [4].

\subsection{Hypergeometric function ${ }_{0} \mathrm{~F}_{1}(; c ; x)$}

The ODE satisfied by the function $y(x)={ }_{0} \mathrm{~F}_{1}(; c ; x)$ is

$$
x^{2} y^{\prime \prime}+c x y^{\prime}-x y=0 .
$$

The solutions of these differential equations have an infinite number of zeros for negative $x$ and are related to Bessel functions:

$$
{ }_{0} \mathrm{~F}_{1}(; c ; z)=\Gamma(c)(-z)^{(1-c) / 2} J_{c-1}(2 \sqrt{-z}) .
$$

\subsubsection{First $D D E$}

Let us consider the DDEs for the family of functions $y_{n}={ }_{0} \mathrm{~F}_{1}(; \gamma+n ;-x)$. The DDEs for this family reads:

$$
\begin{aligned}
& y_{n}^{\prime}=-\frac{c-1}{x} y_{n-1}+\frac{c-1}{x} y_{n}, \\
& y_{n-1}^{\prime}=-\frac{1}{c-1} y_{n},
\end{aligned}
$$

where $c=\gamma+n$. 
The relation with Bessel functions can be expressed saying that, if $y(x)$ is a solution of $x^{2} y^{\prime \prime}+(v+1) x y^{\prime}+x y=0$ then

$$
w(x)=x^{v} y\left(\frac{x^{2}}{4}\right)
$$

is a solution of the Bessel equation $x^{2} w^{\prime \prime}+x w^{\prime}+\left(x^{2}-v^{2}\right) w=0$.

Transforming the DDEs (16) as described in [3], and in section 2, the relevant functions are:

$$
\eta(z(x))=\frac{v-1 / 2}{2 \sqrt{x}}, \quad \tilde{\mathrm{A}}_{n}=1-\frac{v^{2}-1 / 4}{4 x}, \quad z(x)=2 \sqrt{x} .
$$

The fixed point method deriving from this set of DDEs will have identical performance as the system considered in [3], which holds for Ricatti-Bessel functions $j_{v}(x)=\sqrt{x} J_{v}(x)$, that are solutions of the second order ODEs $y^{\prime \prime}+A(x) y=0$, with $A(x)=1-\left(v^{2}-1 / 4\right) / x^{2}$. The identification of both methods with the replacement $x \rightarrow x^{2} / 4$ (according to equation (17) and to the change of variable $z(x)$ ) is evident by comparing the $A(x)$ and $\tilde{\mathrm{A}}_{n}(x)$ coefficients. This is not surprising given that both methods compare the same problem function, $J_{v}(x)$, with the same contrast function, $J_{v-1}(x)$, up to factors which do not vanish (for example, the factor $\sqrt{x}$ for Ricatti-Bessel functions) and up to changes of variable.

\subsubsection{Second $D D E$}

For this type of hypergeometric functions, the only alternative DDEs that can be built are those based on the family of functions $y_{n}={ }_{0} \mathrm{~F}_{1}(; \gamma+m n ;-x) ; m=1$ corresponds to the DDEs (16). We will only consider the case $m=2$ (equivalent, in the sense described before, to $m=-2$ ). For $|m|>2$, the DDEs violate the first imposed condition on the continuity of the coefficients. For the functions $y_{n}={ }_{0} \mathrm{~F}_{1}(; \gamma+2 n ;-x)$, the associated DDEs read:

$$
\begin{aligned}
& y_{n}^{\prime}=-\frac{(c-2)^{2}+(c-2)-x}{(c-2) x} y_{n}+\frac{c-1}{x} y_{n-1}, \\
& y_{n-1}^{\prime}=-\frac{1}{c-2} y_{n-1}-\frac{x}{(c-1)(c-2)^{2}} y_{n},
\end{aligned}
$$

where $c=\gamma+2 n$. The relevant functions in this case are (again, writing $v=c-1$ ):

$$
\begin{aligned}
& \eta(z(x))=\frac{(v-1)^{2}}{2 x}-1, \\
& \tilde{\mathrm{A}}_{n}(z(x))=\left(\frac{v-1}{2 x}\right)^{2}\left(4 x-\left(v^{2}-1\right)\right), \\
& z(x)=\frac{x}{v-1}
\end{aligned}
$$




\subsubsection{Comparison between DDEs}

The second DDE is no longer equivalent to the first one; in fact, it has quite different characteristics. Let us compare the expected performance of these two DDEs, according to the different criteria described above.

To begin with, the $\eta(x)$ parameter never vanishes for the first DDE (DDE1), except when $v=1 / 2$, in which case the method with improved steps is exact without the need to iterate the FPI even once (forward or backward sweeps [7] are used depending on the sign of $\eta(x))$. In contrast, DDE2 has an $\eta$-function which changes sign at $x_{\eta}=(v-1)^{2} / 2$ and the zeros are computed by an expansive sweep [7]. Close to $x_{\eta}$ we can expect that DDE2 tends to behave better in relation to local convergence, because the asymptotic error constant tends to be small.

We observe that, as $x \rightarrow+\infty, \eta(x)$ goes to zero for DDE1 but it tends to -1 for DDE2. This suggests that DDE1 will have faster local convergence than DDE2 for large $x$. On the other hand, as $v$ increases, $\eta(x)$ becomes larger, however, it is difficult to quantify the impact on local converge because as $v$ increases also the smallest zero becomes larger. Let us also take into account that DDE2 will have small $\eta(x)$ for $x$ close to $x_{\eta}$, which becomes large for large $v$.

Regarding the behavior of $\tilde{\mathrm{A}}_{n}(z)$, it is monotonic for DDE1 and has a maximum for DDE2, which allows the use of improved iteration steps. For DDE1, $\tilde{\mathrm{A}}_{n}(z)$ is constant when $v=1 / 2$, which means that sweep with improved iteration steps is exact, as commented before. The maximum for DDE2 is at $x_{m}=\left(v^{2}-1\right) / 2$, where $\tilde{\mathrm{A}}_{n}\left(x_{m}\right)=(v-1) /(v+1)$. Around this extremum, the improved iteration steps tend to work better because $\tilde{\mathrm{A}}_{n}(x)$ will be approximately constant; how constant $\tilde{\mathrm{A}}_{n}(x)$ is around $x_{m}$ can be measured by the convexity at this point. We find:

$$
\ddot{\tilde{\mathrm{A}}}_{n}\left(z_{m}\right)=-8 \frac{v-1}{(v+1)^{3}},
$$

where $z_{m}=z\left(x_{m}\right)$. As $v$ becomes larger, $\ddot{\tilde{A}}_{n}(z)$ becomes smaller around the maximum of $\tilde{\mathrm{A}}_{n}(z)$ and the improved iteration will work better. This fact again, favors DDE2 for large $v$.

Finally, considering the criterion of smaller $D_{n}=\left|d_{n} e_{n}\right|$, we find that, for DDE1

$$
D_{n}=\frac{1}{x}
$$

while for DDE2

$$
D_{n}=\frac{1}{(v-1)^{2}}
$$

This again shows that DDE1 will improve as $x$ increases while DDE2 will be better for large $v$. Numerical experiments show that for $v>100$ the second DDE is preferable over the first, particularly for computing the smallest zeros.

The different criteria yield basically the same information. However the prescription on $D_{n}=\left|d_{n} e_{n}\right|$ is the simplest one to apply. From now on, we will not repeat the 


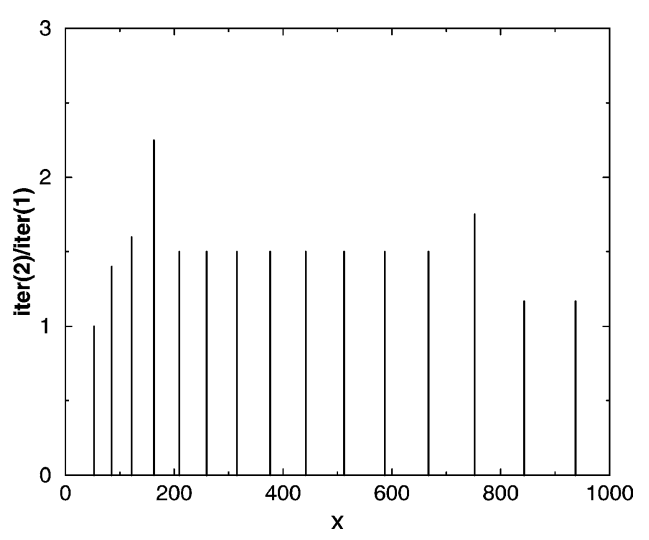

(a)

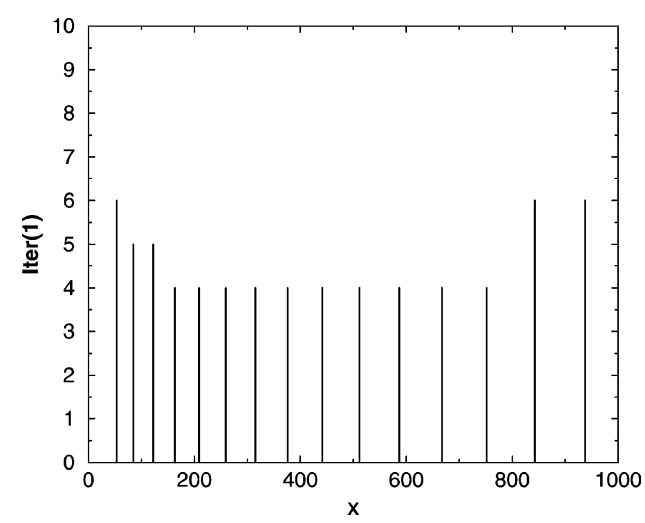

(b)

Figure 1. (a) Ratio between the number of iterations needed for the second and first DDEs for the computation of the zeros of ${ }_{0} \mathrm{~F}_{1}(; 11,-x)$ (the zeros of $J_{10}(2 \sqrt{x})$ ). (b) Number of iterations needed for the first DDE.

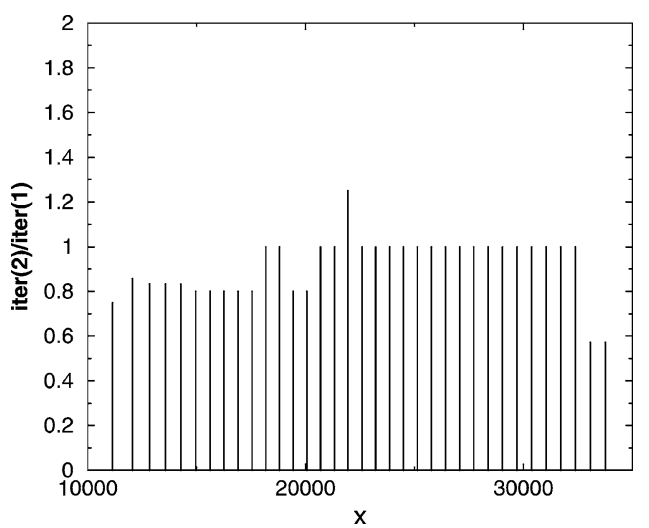

(a)

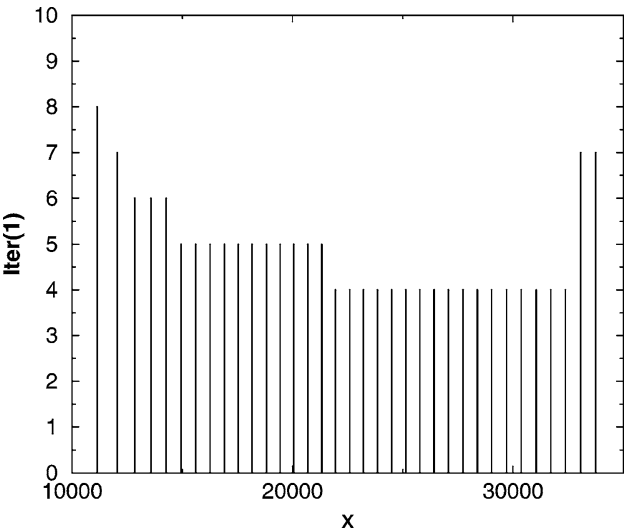

(b)

Figure 2. (a) Ratio between the number of iterations needed for the second and first DDEs for the computation of the zeros of ${ }_{0} \mathrm{~F}_{1}(; 201,-x)$ (the zeros of $J_{200}(2 \sqrt{x})$ ). (b) Number of iterations needed for the first DDE.

analysis for the different criteria. Instead, we adopt this last criterion to analyze the rest of cases.

\subsection{Confluent hypergeometric function}

For the confluent hypergeometric case we have a larger variety of DDEs to choose, because we can choose families of functions $y_{n}={ }_{1} \mathrm{~F}_{1}(a+k n ; c+m n ; x)$, or, more generally, $y_{n}=\phi(a+k n ; c+m n ; x)$, being $\phi$ any solution of equation (13). The families which give rise to DDEs satisfying all our requirements are three, corresponding to the following selections of $(k, m):(1,0),(1,1)$ and $(0,-1)$. 
We will give the corresponding DDEs and the associated functions. At the same time, we will restrict the range of parameters for which the functions are oscillatory (considering the first oscillatory condition in theorem 2.2).

We can restrict the study to $x>0$ because, if $\phi(a ; c ; x)$ is a solution of equation (13), then $e^{x} \phi(c-a ; c ;-x)$ is also a solution of equation (13).

3.2.1. $(k, m)=(1,0) \rightarrow y_{n}=\phi(\alpha+n ; \gamma ; x)$

Let us write, for shortness and in order to compare with other recurrences $a=$ $\alpha+n, c=\gamma$. As before commented, we consider simultaneously the equivalent directions $(k, m)=(1,0)$ and $(k, m)=(-1,0)$ but we present only the DDEs and related functions for the recurrence direction for which the FPI based on the ratio $H_{-1}=y_{n} / y_{n-1}$ can be used with improved iteration steps (theorem 2.1); this is the direction $(1,0)$.

The $(-1,0)$ direction is the natural one for orthogonal polynomials of hypergeometric type (Laguerre, Hermite), which are related to confluent hypergeometric series of the type ${ }_{1} \mathrm{~F}_{1}(-n ; \gamma ; x)$ (see, for instance, [6, equations (9.13.8)-(10)]).

The DDEs for $(k, m)=(1,0)$ read

$$
y_{n}^{\prime}=\frac{a-c+x}{x} y_{n}-\frac{a-c}{x} y_{n-1}, \quad y_{n-1}^{\prime}=-\frac{a-1}{x} y_{n-1}+\frac{a-1}{x} y_{n}
$$

and therefore, applying the first oscillatory condition of theorem 2.2, the parameters are restricted to:

$$
(a-c)(a-1)>0
$$

otherwise the functions $y_{n}, y_{n-1}$ will be non-oscillatory. If we repeat the same argument for $(k, m)=(-1,0)$ or, equivalently, apply the same criteria for the DDEs for the functions $y_{n}, y_{n+1}$ the following restriction is obtained:

$$
(a-c+1) a>0 .
$$

The associated functions for this DDE are

$$
\begin{aligned}
& \eta(z(x))=-\frac{2 a+c+x}{2 \sqrt{(c-a)(1-a)}} \\
& \tilde{\mathrm{A}}_{n}(z(x))=\frac{-x^{2}+2(c-2 a) x-(c-1)^{2}}{4(c-a)(1-a)} \\
& z(x)=\sqrt{(c-a)(1-a)} \ln x
\end{aligned}
$$

Let us notice that $\eta(x)$ becomes negative for large $x$, which gives the most appropriate sweep for large $x$ (forward) since $\tilde{\mathrm{A}}_{n}(z(x))$ decreases for large $x$.

Observe that the lack of a singularity in $\tilde{\mathrm{A}}_{n}(z(x))$ is only apparent because the function $z(x)$ is singular at $x=0$. 
3.2.2. $(k, m)=(0,-1) \rightarrow y_{n}=\phi(\alpha ; \gamma-n ; x)$

As before, we denote $a=\alpha, c=\gamma-n$. The DDEs for the system read:

$$
\begin{aligned}
& y_{n}^{\prime}=y_{n}+\frac{a-c}{c} y_{n-1}, \\
& y_{n-1}^{\prime}=-\frac{c}{x} y_{n-1}+\frac{c}{x} y_{n}
\end{aligned}
$$

which implies the restriction $(c-a) x>0$ for the solutions to have oscillatory nature. Repeating the same for $(k, m)=(0,1)$, we arrive at the condition $(c-a-1) x>0$, which for $x>0$ gives a more restrictive condition $c-a>1$ (for $x<0$, the first condition is more restrictive and gives $a-c<0$ ).

Let us recall that the condition $c-a>1$ for $x>0$ means that, if this condition is not met, neither $y_{n}$ nor $y_{n-1}$ can have two zeros in $x>0$. Given that we are interested in computing zeros of oscillatory functions, we consider these restrictions. Let us however notice that the possible isolated zero of $y_{n}$ for $x>0$ and $0<c-a<1$, could be also computed by means of the FPM associated to the DDEs (25).

Considering also the restrictions imposed in the previous selection of DDEs, we obtain the following:

Theorem 3.1. Let $y$ be a solution of the confluent hypergeometric equation (13) for $x>0$. If $y$ has at least two zeros then $c-a>1$ and $a<0$.

Let $y$ be a solution of the confluent hypergeometric equation (13) for $x<0$. If $y$ has at least two zeros then $c-a<0$ and $a>1$.

For more detailed results on the number of zeros of confluent hypergeometric functions, we refer the reader to [2, Vol. 1, section 6.16].

The associated functions are

$$
\begin{aligned}
& \eta(z(x))=-\frac{2 c-1+2 x}{4 \sqrt{(c-a) x}} \\
& \tilde{\mathrm{A}}_{n}(z(x))=\frac{8 c x-16 x a-3+8 c-4 c^{2}-4 x^{2}}{16(c-a) x} \\
& z=2 \sqrt{(c-a) x}
\end{aligned}
$$

Let us notice that $\eta(z(x))$ becomes negative for large $x$, which gives the most appropriate sweep for large $x$ (forward) since, for positive $x, \tilde{\mathrm{A}}_{n}(z(x))$ decreases for large $x$.

3.2.3. $(k, m)=(1,1) \rightarrow y_{n}=\phi(\alpha+n ; \gamma+n ; x)$

The DDEs are the following (writing $a=\alpha+n, c=\gamma+n$ ).

$$
\begin{aligned}
& y_{n}^{\prime}=\frac{x+1-c}{x} y_{n}+\frac{c-1}{x} y_{n-1}, \\
& y_{n-1}^{\prime}=\frac{a-1}{c-1} y_{n}
\end{aligned}
$$


which implies the oscillatory condition $(a-1) x>0$; considering $(k, m)=(-1,-1)$ or, equivalently, the DDEs relating $y_{n}, y_{n+1}$ and their derivatives, the condition obtained is $a x>0$. These conditions are consistent with theorem 3.1.

The functions associated to these DDEs for $x>0$ are

$$
\begin{aligned}
& \eta=-\frac{2 x+3-2 c}{4 \sqrt{(1-a) x}}, \\
& z=2 \sqrt{(1-a) x}, \\
& \tilde{\mathrm{A}}_{n}(z(x))=\frac{16 x a+4 x^{2}-8 x c+3-8 c+4 c^{2}}{16(-1+a) x} .
\end{aligned}
$$

This iteration cannot be used for $c=1$ (see equation (27)) unless the FPI stemming from the ratio $y_{n} / y_{n+1}$ is used, in which case the improved iteration steps cannot be applied.

\subsubsection{Comparing fixed point iterations}

As commented before, we will consider the prescription consisting in choosing the DDEs for which the product $D_{n}=-d_{n} e_{n}$ is smaller. Applying literally this criterion, we find the following preferred regions of application:

1. FP(1,1) should be applied for $x<c-a$ and $\mathrm{FP}(1,0)$ for $x>c-a$.

2. $\mathrm{FP}(1,1)$ is always better than $\mathrm{FP}(0,-1)$.

3. $\mathrm{FP}(0,-1)$ is better than $\mathrm{FP}(1,0)$ for $x<1-a$, but $1-a<c-a$ and $\mathrm{FP}(1,1)$ is better there.

Therefore, the best combination of the considered FPIs is $\mathrm{FP}(1,1)$ for $x<c-a$ and $\mathrm{FP}(1,0)$ for $x>c-a$. The iteration $\mathrm{FP}(0,-1)$ has the same behavior as $\mathrm{FP}(1,1)$ and can be used as a replacement when $c=1$ (in this case $\mathrm{FP}(1,1)$ can not be used). In fact, the $\mathrm{FP}(0,-1)$ and $\mathrm{FP}(1,1)$ are not independent because, as it is well known, if $\psi(\alpha ; \gamma ; x)$ are solutions of the confluent hypergeometric equation $x \psi^{\prime \prime}+(\gamma-x) \psi^{\prime}-\alpha \psi=0$, then $y(a ; c ; x)=x^{1-c} \psi(1+a+c ; 2-c ; x)$ is a solution of $x y^{\prime \prime}+(c-x) y^{\prime}-a y=0$.

Let us illustrate this behavior with numerical examples:

In figure 3 we compare $\mathrm{FP}(1,1)$ with $\mathrm{FP}(1,0)$ for the case of Laguerre polynomials $L_{n}^{\alpha}(x)$ for the quite extreme case $n=50, \alpha=-0.9999$. On the left, the ratio between the number of iterations employed by $\operatorname{FP}(1,1)$ and $\operatorname{FP}(1,0)$ is shown as a function of the location of the zeros. The first two zeros are skipped in figure 3(a) to show in more detail the behavior for large $x$ (for the first zero the ratio was 40 while for the second it was 5). The improvement for small $x$ when considering $\operatorname{FP}(1,1)$ is quite noticeable. For larger $x$ $(x>c-a \simeq 50), \operatorname{FP}(1,0)$ works generally better than $\mathrm{FP}(1,1)$ but the improvement is not so noticeable. In figure 3(b), the number of iterations used to compute each zero when considering $\mathrm{FP}(1,1)$ is shown.

In conclusion, $\mathrm{FP}(1,1)$ is a more appropriate choice than the natural recurrence for orthogonal polynomials $(\mathrm{FP}(1,0))$, although $\mathrm{FP}(1,0)$ slightly improves the convergence of $\mathrm{FP}(1,1)$ for $x>c-a$. 


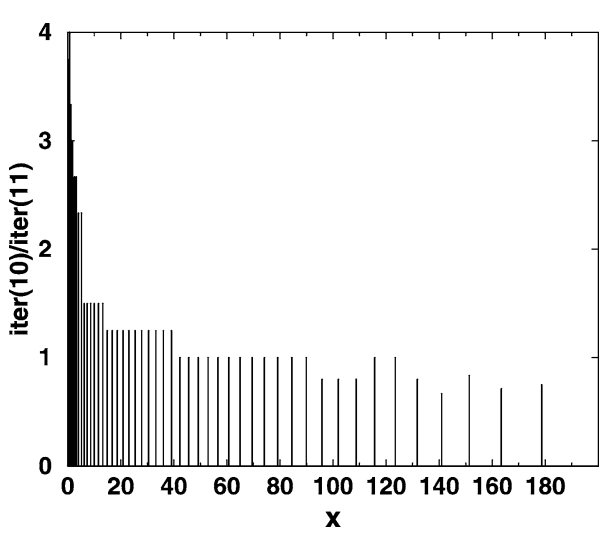

(a)

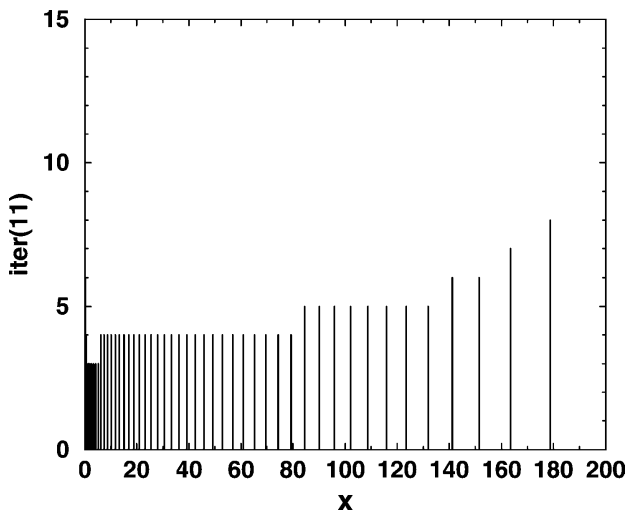

(b)

Figure 3. (a) Ratio between the number of iterations needed by $\operatorname{FP}(1,0)$ and $\operatorname{FP}(1,1)$ for the calculation of the zeros of the generalized Laguerre polynomial $L_{50}^{-0.9999}(x)$, as a function of the location of the zeros. (b) Number of iterations needed by FP(1,1).

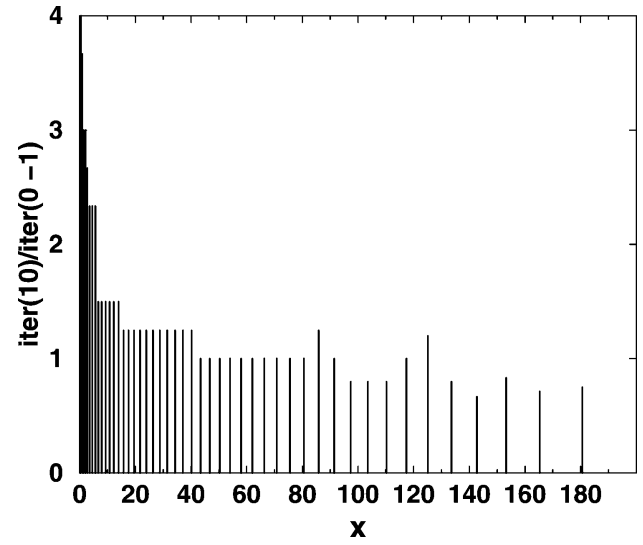

(a)

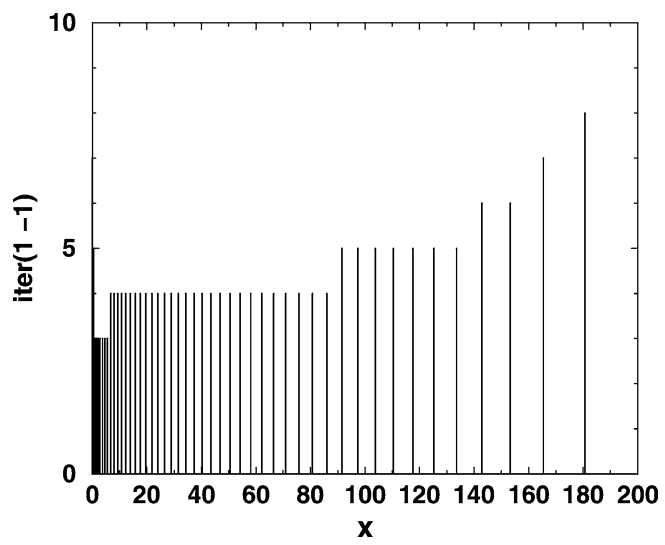

(b)

Figure 4. (a) Ratio between the number of iterations needed by $\operatorname{FP}(1,0)$ and $\operatorname{FP}(0,-1)$ for the calculation of the zeros of the generalized Laguerre polynomial $L_{50}^{0}(x)$, as a function of the location of the zeros. (b) Number of iterations needed by $\operatorname{FP}(0,-1)$.

In figure 4 we compare $\mathrm{FP}(1,0)$ with $\mathrm{FP}(0,-1)$ for the case of generalized Laguerre polynomials but now for a choice of the parameters $n=50, \alpha=0$. This situation corresponds to the case $c=1$, where $\operatorname{FP}(1,1)$ can not be applied. As in figure 3 , the ratio of the number of iterations for the first two zeros is not plotted (the ratio of the first zero was 8 and for the second it was 5). As expected from our previous analysis, the iteration $\mathrm{FP}(0,-1)$ behaves quite better than $\mathrm{FP}(1,0)$ for small $x$.

\subsection{Mysterious hypergeometric function}

This is the name given to the hypergeometric series ${ }_{2} \mathrm{~F}_{0}(a, b ; ; x)$, which diverges for all $x \neq 0$ (except in the terminating cases) and can only be interpreted in an asymp- 
totic sense. It is well know that, for negative $x$ we have:

$$
{ }_{2} \mathrm{~F}_{0}(a, b ; ; x)=\left(\frac{-1}{x}\right)^{a} U\left(a, 1+a-b, \frac{-1}{x}\right),
$$

where $U(a, c, x)$ is a solution of the confluent hypergeometric equation (13). The functions ${ }_{2} \mathrm{~F}_{0}$ can be analytically continued to the whole complex plane cut along the line $\Re(z)>1, \Im(z)=0$. The function (29) is a solution of the $2-0$ hypergeometric differential equation:

$$
x^{2} y^{\prime \prime}+[-1+x(a+b+1)] y^{\prime}+a b y=0 .
$$

In general terms, without referring to any particular solution of the corresponding differential equations, the problem of computing the real zeros of the mysterious hypergeometric function for negative $x$ can be transformed into a problem of computation of the zeros of confluent hypergeometric functions equation (29). This is so because one can check that, if we denote by $y(\alpha, \beta, x)$ a set of solutions of the confluent hypergeometric equation, then $w(x)=|x|^{-a} y(a, 1+a-b,-1 / x)$, for $x>0$ or $x<0$, are solutions of equation (30).

For this reason and for brevity we omit further details.

\subsection{Gauss hypergeometric functions}

Let us consider the hypergeometric function ${ }_{2} \mathrm{~F}_{1}(a, b ; c ; x)$. We will consider the DDEs for families of functions of the type $\psi(\alpha+k n, \beta+m n ; \gamma+\ln ; x)$, with $\psi(a, b ; c ; x)$ solutions of the hypergeometric equation (14); we use the DDEs for ${ }_{2} \mathrm{~F}_{1}(a, b ; c ; x)$ series as generated by hsum.mpl.

Similarly as we did for the confluent case, we can obtain oscillatory conditions for the coefficients $a, b$ and $c$, depending on the range of $x$. If these conditions are not satisfied by the parameters, then we can assure that if there exists one zero of the function, this is an isolated zero. As before, these conditions are obtained by requiring that $d_{n} e_{n}<0$; combining the restrictions imposed by this condition for the DDEs that we will later show we obtain the following:

Theorem 3.2. Let $\psi(a, b ; c ; x)$ be a solution of equation (14) defined in $(-\infty, 0)$, then if this function is oscillatory in this interval (it has at least two zeros) then one of the following sets of conditions must be verified:

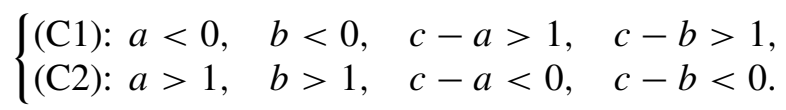

Similarly, if $\psi(a, b ; c ; x)$ is a solution of equation (14) defined in $(0,1)$, the oscillatory conditions are

$$
\begin{cases}(\mathrm{C} 3): & a<0, \quad b>1, \quad c-a>1, \quad c-b<0, \\ \text { (C4): } a>1, \quad b<0, & c-a<0, \quad c-b>1 .\end{cases}
$$


Finally, if $\psi(a, b ; c ; x)$ is a solution of equation (14) defined in $(1,+\infty)$ and is oscillatory in this interval, one of the following sets of conditions must be verified:

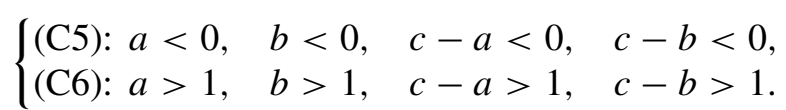

The different sets of conditions for the three subintervals in theorem 3.2 can be obtained by combining the restrictions obtained from the following values of $(k, l, m)$ : $( \pm 1,0,0),( \pm 1, \pm 1,0),( \pm 1,0, \pm 1),( \pm 1, \mp 1,0),(0,0, \pm 1)$ and $( \pm 1, \pm 1, \pm 1)$. In any case, the analysis for the three different subintervals are not independent because, as it is well know (see [2, Vol. I, chapter II]), if we denote by $\psi(\alpha, \beta ; \gamma, x)$ the solutions of the hypergeometric equation $x(1-x) y^{\prime \prime}+(\gamma-(\alpha+\beta+1) x) y^{\prime}-\alpha \beta y=0$ in the interval $(0,1)$ one can write solutions in the other two intervals by using that both

$$
y(a, b ; c ; x)=(1-x)^{-a} \psi\left(a, c-b ; c ; \frac{x}{x-1}\right), \quad x<0
$$

and

$$
y(a, b ; c ; x)=x^{-a} \psi\left(a, a+1-c ; a+b+1-c ; 1-\frac{1}{x}\right), \quad x>1
$$

are solutions of the hypergeometric differential equation $x(1-x) y^{\prime \prime}+(c-(a+$ $b+1) x) y^{\prime}-a b y=0$. With this, it is easy to see that the conditions $\mathrm{C} 1$ and $\mathrm{C} 2$ can be obtained from $\mathrm{C} 3$ and $\mathrm{C} 4$, respectively, by using equation (31), while $\mathrm{C} 5$ and $\mathrm{C} 6$ derive from $\mathrm{C} 3, \mathrm{C} 4$ and equation (32).

Notice, in addition, that the 6 conditions in theorem 3.2 are mutually exclusive, which means that:

Theorem 3.3. Given three values of the parameters $a, b$ and $c$, at most one of the subintervals $(-\infty, 0),(0,1),(1,+\infty)$ possesses oscillatory solutions (solutions with at least two zeros).

The oscillatory conditions $\mathrm{C} 6$ is of no use for non-terminating hypergeometric series, because they diverge for $x>1$; however, this is a possible case for other solutions of the differential equation. The conditions C 3 correspond to Jacobi polynomials

$$
P_{n}^{(\alpha, \beta)}(x)=\frac{(\alpha+1)_{n}}{n !}{ }_{2} \mathrm{~F}_{1}\left(-n, 1+\alpha+\beta+n ; \alpha+1 ; \frac{1-x}{2}\right), \quad \alpha, \beta>-1
$$

of order $n \geqslant 2$ (for order $n=1$ the conditions are not satisfied, not surprisingly because according to our criteria a polynomial of degree 1 is non-oscillating). Particular cases of Jacobi polynomials are Gegenbauer $(\alpha=\beta)$, Legendre $(\alpha=\beta=0)$ and Chebyshev $(\alpha=\beta=-1 / 2)$ polynomials. 


\subsubsection{DDEs and change of variables}

In this section, we compile the expressions for the different DDEs as well as the associated change of variable. For brevity, the associated functions $\eta(x)$ and $\tilde{\mathrm{A}}_{n}(x)$ are not shown.

It is understood that $a=\alpha+k n, b=\beta+l n, c=\gamma+m n$ for $\operatorname{DDE}(k, l, m)$.

1. $\operatorname{DDE}(1,0,0)$

$$
y_{n}^{\prime}=\frac{-a-b x+c}{x(x-1)} y_{n}+\frac{a-c}{x(x-1)} y_{n-1}, \quad y_{n-1}^{\prime}=\frac{1-a}{x} y_{n-1}+\frac{a-1}{x} y_{n} .
$$

Change of variable: $z(x)=-2 \sqrt{(c-a)(1-a)} \tanh ^{-1}(\sqrt{1-x})$.

2. $\operatorname{DDE}(1,1,0)$ :

$$
\begin{aligned}
y_{n}^{\prime}= & \left(\frac{-(a+b)(a+b-c-1)+a b-c}{x(a+b-c-1)}+\frac{a+b-c}{x(1-x)}\right) y_{n} \\
& +\frac{(a-c)(c-b)}{x(1-x)(a+b-c-1)} y_{n-1}, \\
y_{n-1}^{\prime}= & \frac{a+b-a b-1}{(a+b-c-1) x} y_{n-1}-\frac{(1-x)(a-1)(1-b)}{(a+b-c-1) x} y_{n} .
\end{aligned}
$$

Change of variable:

$$
z(x)=\frac{\sqrt{(b-c)(c-a)(b-1)(1-a)}}{|a+b-c-1|} \ln x, \quad x>0 .
$$

3. $\operatorname{DDE}(1,1,2)$ :

$$
\begin{aligned}
y_{n}^{\prime}= & \frac{(1-x)[(1-a-b)(c-1)+a b]+(c-1)(1+a+b-c)-a b}{x(1-x)(c-2)} y_{n} \\
& -\frac{(1-c)}{x(1-x)} y_{n-1}, \\
y_{n-1}^{\prime}= & \frac{1-a-b+a b}{(1-x)(c-2)} y_{n-1}-\frac{x(a-c+1)(1-a)(c-b-1)(b-1)}{(1-x)(c-1)(c-2)^{2}} y_{n} .
\end{aligned}
$$

Change of variable:

$$
z(x)=-\sqrt{\frac{(c-a-1)(1-a)(1+b-c)(b-1)}{(c-2)^{2}}} \ln (1-x) .
$$

4. $\operatorname{DDE}(1,0,1)$ :

$$
\begin{aligned}
y_{n}^{\prime} & =\frac{1+x b-c}{x(1-x)} y_{n}-\frac{1-c}{x(1-x)} y_{n-1}, \\
y_{n-1}^{\prime} & =-\frac{(1-a)}{(1-x)} y_{n-1}+\frac{(1-a)(b+1-c)}{(1-x)(1-c)} y_{n} .
\end{aligned}
$$


Change of variable: $z(x)=2 \sqrt{(1-a)(b+1-c)} \tanh ^{-1} \sqrt{x}$.

5. $\operatorname{DDE}(1,-1,0)$ :

$$
\begin{aligned}
y_{n}^{\prime} & =b \frac{x(b-a+1)+a-c}{x(1-x)(b-a+1)} y_{n}+\frac{b(c-a)}{x(1-x)(b-a+1)} y_{n-1}, \\
y_{n-1}^{\prime} & =(1-a) \frac{(1-x)(b-a+1)+a-c}{x(1-x)(b-a+1)} y_{n-1}-\frac{(1-a)(1+b-c)}{x(1-x)(b-a+1)} y_{n} .
\end{aligned}
$$

Change of variable:

$$
z(x)=\frac{\sqrt{b(c-a)(1-a)(1+b-c)}}{b-a+1} \ln \left(\frac{x}{1-x}\right) .
$$

6. $\operatorname{DDE}(0,0,-1)$ :

$$
\begin{aligned}
y_{n}^{\prime} & =\frac{b+a-c}{1-x} y_{n}-\frac{(b-c)(c-a)}{(1-x) c} y_{n-1}, \\
y_{n-1}^{\prime} & =-\frac{c}{x} y_{n-1}+\frac{c}{x} y_{n} .
\end{aligned}
$$

Change of variable: $z(x)=\sqrt{(b-c)(c-a)} \arcsin (2 x-1)$.

7. $\operatorname{DDE}(1,1,1)$ :

$$
\begin{aligned}
y_{n}^{\prime} & =\frac{x(a+b-1)-c+1}{x(1-x)} y_{n}-\frac{1-c}{x(1-x)} y_{n-1}, \\
y_{n-1}^{\prime} & =\frac{(b-1)(1-a)}{(1-c)} y_{n} .
\end{aligned}
$$

Change of variable: $z(x)=\sqrt{(b-1)(1-a)} \arcsin (2 x-1)$.

\subsubsection{Comparison of FPIs}

Table 1 shows the $\left|D_{n}\right|=\left|-d_{n} e_{n}\right|$ coefficient for the FPIs $(1,0,0),(1,1,0)$, $(1,1,2),(1,0,1),(1,-1,0),(0,0,-1)$ and $(1,1,1)$.

As can be inferred from the table, the most appropriate FPIs in the interval $(0,1)$ are the $(0,0,-1)$ and $(1,1,1)$ iterations. As commented, some hypergeometric functions in this interval with particular values of their parameters are orthogonal polynomials (Jacobi and derived polynomials). In the case of orthogonal polynomials, the "natural" iteration to be considered is $(1,-1,0)$ (or $(-1,1,0)$ equivalently) which is not the optimal iteration. In order to illustrate this fact, let us consider the evaluation of the zeros of the hypergeometric function ${ }_{2} \mathrm{~F}_{1}(-50,54 ; 5 / 2 ; x)$ in the interval $(0,1)$. The zeros of this function correspond to the zeros of the Jacobi polynomial $P_{50}^{(3 / 2,3 / 2)}(1-2 x)$. In figure 5 , we show the ratio between the number of iterations needed by $\operatorname{FP}(1,-1,0)$ and $\mathrm{FP}(1,1,1)$ as a function of the location of the zeros.

On the contrary, the most appropriate iteration in the $(1, \infty)$ interval is the $(1,0,0)$ iteration. This could also be understood taking into account that the best iterations in the 
Table 1

\begin{tabular}{ll}
\hline Iteration & \multicolumn{1}{c}{$\left|D_{n}\right|$} \\
\hline$(1,0,0)$ & $\left|\frac{(a-c)(a-1)}{x^{2}(1-x)}\right|$ \\
$(1,1,0)$ & $\left|\frac{(b-c)(a-c)(b-1)(a-1)}{x^{2}(a+b-c-1)^{2}}\right|$ \\
$(1,1,2)$ & $\left|\frac{(c-a-1)(1-a)(1+b-c)(b-1)}{(1-x)^{2}(c-2)^{2}}\right|$ \\
$(1,0,1)$ & $\left|\frac{(a-1)(1+b-c)}{x(1-x)^{2}}\right|$ \\
$(1,-1,0)$ & $\left|\frac{b(c-a)(a-1)(c-b-1)}{x^{2}(1-x)^{2}(a-b-1)^{2}}\right|$ \\
$(0,0,-1)$ & $\left|\frac{(b-c)(a-c)}{x(1-x)}\right|$ \\
$(1,1,1)$ & $\left|\frac{(b-1)(a-1)}{x(1-x)}\right|$ \\
\hline
\end{tabular}

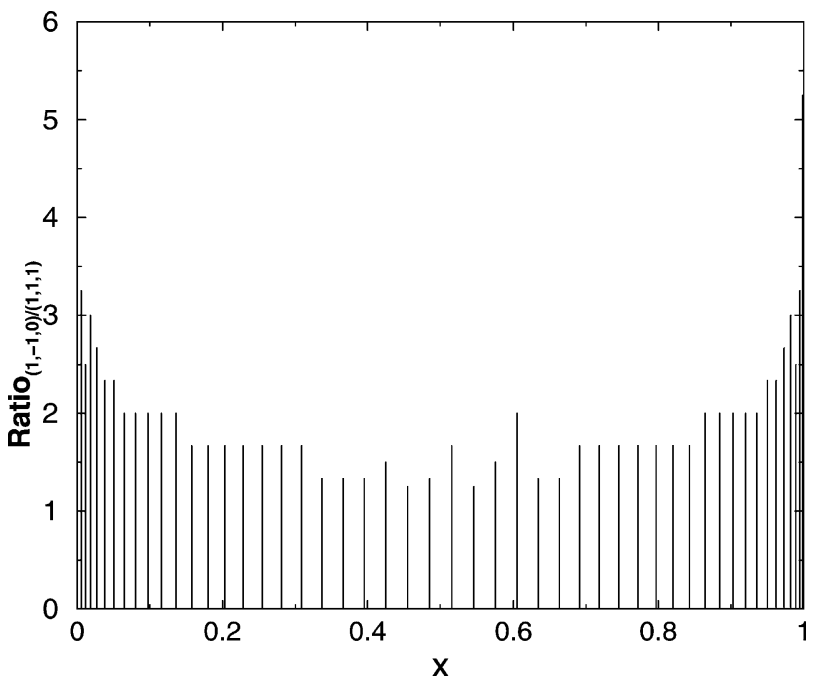

Figure 5. Ratio between the number of iterations needed by $\operatorname{FP}(1,-1,0)$ and $\operatorname{FP}(1,1,1)$ for the calculation of the zeros of the hypergeometric function ${ }_{2} \mathrm{~F}_{1}(-50,54 ; 5 / 2 ; x)$, as a function of the location of the zeros.

interval $(0,1)$ are $\operatorname{FPI}(0,0,-1)$ and $\operatorname{FPI}(1,1,1)$ and using equation (32). In figure 6 we show the ratio between the number of iterations needed by $\operatorname{FP}(1,1,1)$ and $\operatorname{FP}(1,0,0)$.

The main conclusion for ${ }_{2} \mathrm{~F}_{1}$ hypergeometric functions is that the iteration $(1,1,1)$ is the preferred one and that $(0,0,-1)$ can be considered as a replacement, with similar performance. For the other two intervals, relations (31) and (32) indicate that the most appropriate iterations will be $(1,0,1)$ for $(-\infty, 0)$ and $(1,0,0)$ for $(1,+\infty)$; in any case, the solutions in these intervals can be related to solutions in $(0,1)$. 


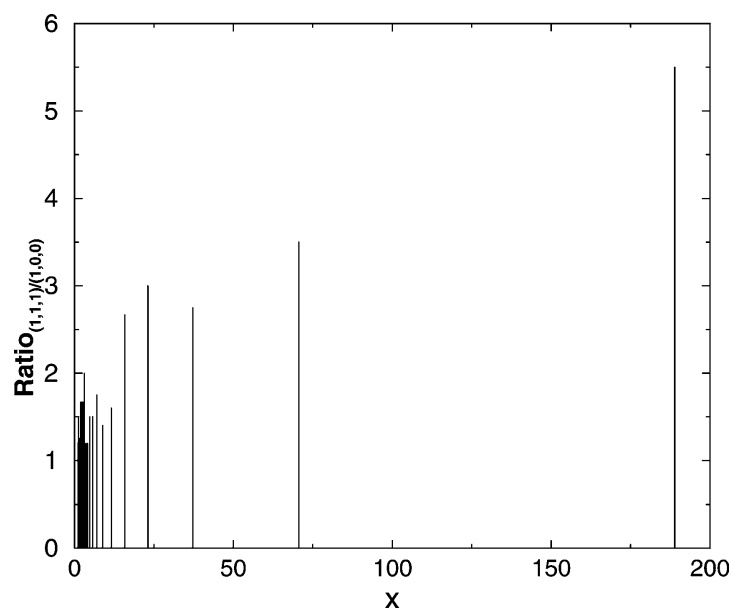

Figure 6. Ratio between the number of iterations needed by $\operatorname{FP}(1,1,1)$ and $\operatorname{FP}(1,0,0)$ for the calculation of the zeros of the hypergeometric function ${ }_{2} \mathrm{~F}_{1}(-30,-32 ;-70 ; x)$, as a function of the location of the zeros.

Table 2

\begin{tabular}{clll}
\hline \multicolumn{1}{c}{ Function } & Contrast function & Range of application & Change of variables $z(x)$ \\
\hline${ }_{0} \mathrm{~F}_{1}(; c ;-x)$ & ${ }_{0} \mathrm{~F}_{1}\left(; c_{-} ;-x\right)$ & $\left\{\begin{array}{l}c<100 \\
c>100, x>c^{2} / 2\end{array}\right.$ & $2 \sqrt{x}$ \\
& ${ }_{0} \mathrm{~F}_{1}(; c-;-x)$ & $c>100, x<c^{2} / 2$ & $x /(v-1)$ \\
${ }_{1} \mathrm{~F}_{1}(a ; c ; x)$ & ${ }_{1} \mathrm{~F}_{1}\left(a_{-} ; c c_{-} ; x\right)$ & $x<c-a$ & $2 \sqrt{(1-a)} x$ \\
& ${ }_{1} \mathrm{~F}_{1}\left(a_{-} ; c ; x\right)$ & $x>c-a$ & $N_{a c} \ln x$
\end{tabular}

\section{Conclusions}

We have developed a detailed study of the performance of the available fixed point methods for hypergeometric functions. This will allow the construction of efficient algorithms for the computation of the real zeros of hypergeometric functions with a good asymptotic behavior. Table 2 summarized the main results. In the table we restrict ourselves to $x>0$ in all cases, with the additional restriction $x<1$ for the ${ }_{2} \mathrm{~F}_{1}$ functions. For the rest of the intervals, as commented before, relations are available which map these other regions into the intervals considered in the table.

It should be noted that, when a priori approximations to the roots are available (for instance, asymptotic approximations like in [8]) the performance of the algorithms can be improved. However, the methods presented here have the advantage of being efficient methods that do not require specific approximations for specific functions (which, on the other hand, are difficult to obtain for three parameter functions like the ${ }_{2} \mathrm{~F}_{1}$ hypergeo- 
metric functions). In addition, even in the simple cases of one parameter functions, the methods are very efficient by themselves.

To conclude, it is worth mentioning that one on the main reasons for the good performance of the algorithms if that the analytical transformations of the DDEs, and in particular, the associated change of variable $z(x)=\int \sqrt{-d_{n} e_{n}} \mathrm{~d} x$, tend to uniformize the distance between zeros. Generally speaking, the most successful methods are those which produce smaller variations of the distances between zeros, because the first guesses for the zeros become more accurate. In connection to this, these changes of variable lead to interesting analytical information about these zeros [1].

\section{Acknowledgements}

A. Gil acknowledges support from A. von Humboldt foundation. J. Segura acknowledges support from DAAD.

\section{Appendix. Maple code}

In this appendix we would like to explain how we received the DDEs automatically. Our Maple code uses a Zeilberger-type approach [9] and is completely on the lines of [5]. The authors provide a Maple program rules.mpl which can be used in combination with hsum.mpl [5] to get equations (15), (18), (22), (24), (26), (28), and the DDEs in section 3.4.1. These computations are collected in the Maple worksheet rules.mpl. All these files can be obtained from the Web site http: / /www. mathematik . unikassel.de/ koepf/Publikationen.

\section{References}

[1] A. Deaño, A. Gil and J. Segura, New inequalities from classical Sturm theorems, submitted.

[2] A. Erdélyi, W. Magnus, F. Oberhettinger and F.G. Tricomi, Higher Transcendental Functions (McGraw-Hill, New York, 1953).

[3] A. Gil and J. Segura, Computing zeros and turning points of linear homogeneous second order ODEs, SIAM J. Numer. Anal. 41 (2003) 827-855.

[4] A. Gil and J. Segura, A combined symbolic and numerical algorithm for the computation of zeros of orthogonal polynomials and special functions, J. Symbol. Comp. 35 (2003) 465-485.

[5] W. Koepf, Hypergeometric Summation. An Algorithmic Approach to Summation and Special Function Identities (Vieweg, Braunschweig/Wiesbaden, 1998).

[6] N.N. Lebedev, Special Functions and Their Applications (Dover, New York, 1972).

[7] J. Segura, The zeros of special functions from a fixed point method, SIAM J. Numer. Anal. 40 (2002) 114-133.

[8] N.M. Temme, An algorithm with Algol 60 program for the computation of the zeros of ordinary Bessel functions and those of their derivatives, J. Comput. Phys. 32 (1979) 270-270.

[9] D. Zeilberger, A fast algorithm for proving terminating hypergeometric identities, Discrete Math. 80 (1990) 207-211. 